\title{
Weak turbulence theory of dispersive waves in the solar corona
}

\author{
Felix Spanier ${ }^{1}$ and Rami Vainio ${ }^{2}$ \\ ${ }^{1}$ Lehrstuhl für Astronomie, Universität Würzburg, Am Hubland, 97074 Würzburg, Germany \\ email: fspanier@astro.uni-wuerzburg.de \\ ${ }^{2}$ University of Helsinki, Department of Physics, P.O.Box 64, 00014 University of Helsinki, \\ Finland \\ email: rami.vainio@helsinki.fi
}

\begin{abstract}
The interaction of plasma waves plays a crucial role in the dynamics of weakly turbulent plasmas. So far the interaction of non-dispersive waves has been studied. In this paper the theory is extended to dispersive waves. It is well known that dispersive waves may be found in the solar corona, where they contribute to the heating of the corona. Here the possible interactions in the solar corona are described and the interaction rates are derived in the framework of Hall MHD.
\end{abstract}

Keywords. Sun: corona, plasmas, waves

\section{Introduction}

The sun exhibits a wide range of plasma phenomena from its core to the solar wind. On all scales thermal plasma, turbulent waves and non-thermal particle distributions can be found. Due to their variety not all phenomena can be explained by single model. Here we focus on coronal holes, where the turbulence level is rather low and the weak turbulence model, i.e., the interaction of plasma waves, is an adequate description. Weak turbulence theory allows the calculation of energy transport between different wave modes on different scales. From observation of nonthermal velocity fluctuations of ions (e.g., Laitinen et al., 2003) one can show that magnetic field perturbation amplitudes are well below the background magnetic field, which is necessary prerequisite for the application of weak turbulence theory. We will use this theory to describe the problem of turbulence evolution of coronal holes.

One class of models for the heating of coronal holes is based on the dissipation of low-frequency $\left(\omega<\Omega_{i}\right)$ electromagnetic plasma waves. Such waves could be generated, for example, by small reconnection events in the chromospheric magnetic network below coronal holes (Axford \& McKenzie, 1992). However, the dissipation and spectral evolution of such waves is still not properly understood, as the results of wave-wave interactions and dispersion are typically neglected. In this paper, we attempt to describe the non-linear interaction of dispersive plasma waves in coronal-hole-type plasmas.

\section{Derivation of the wave-wave interaction}

Following the approach from Sagdeev \& Galeev (1969) plasma waves are considered as quasi-particles. Therefore in the interaction of three waves momentum and energy of the waves have to be conserved

$$
\begin{aligned}
& \vec{k}_{\mathrm{M}}=\vec{k}_{\mathrm{P}}+\vec{k}_{\mathrm{Q}} \\
& \omega_{\mathrm{M}}=\omega_{\mathrm{P}}+\omega_{\mathrm{Q}}
\end{aligned}
$$


This system has been studied for Alfvén and sound waves by Chin \& Wentzel (1972) and Vainio \& Spanier (2005). These studies limited to frequencies well below the iongyrofrequency. For dispersive waves the three-wave interactions have been studied by Spanier \& Vainio (2009), where L- and R-modes as well as sound modes are considered. An outcome of this study is also that not all interactions allowed by the resonance condition are actually possible: Additionally in all interactions angular momentum has to be conserved, where $\mathrm{L}$ waves are assigned angular momentum $\hbar$ and $\mathrm{R}$ waves $-\hbar$. One finds in the dispersive regime interactions which are directly linked to non-dispersive waves and also purely dispersive interactions which are found mainly in the ion-gyrofrequency regime.

To derive the interaction rate of dispersive wave modes one may either use the full kinetic theory provided by Melrose \& Sy (1972) or use the ansatz of Chin \& Wentzel in the Hall MHD regime. Since the full kinetic theory is fairly complex (see Yoon \& Fang, 2008) we resorted to the Hall MHD ansatz. Using the set of MHD equations

$$
\begin{aligned}
\partial_{t} \rho+\nabla \cdot(\rho \vec{v}) & =0 \\
\rho \partial_{t} \vec{v}+\rho(\vec{v} \cdot \nabla) \vec{v} & =-\nabla p-\frac{1}{4 \pi} \vec{B} \times(\nabla \times \vec{B}) \\
\partial_{t} \vec{B} & =\nabla \times(\vec{v} \times \vec{B})-\frac{m_{i} c}{4 \pi e} \nabla \times\left(\frac{1}{\rho}(\nabla \times \vec{B}) \times \vec{B}\right) \\
\nabla p & =c_{s}^{2} \nabla \rho
\end{aligned}
$$

one employs perturbation theory to derive the interaction rate. Using the notation of Chin \& Wentzel one finds

$$
\partial_{t} \vec{v}=-\frac{\mathfrak{i}}{2 \omega} \vec{S}\left(\vec{v}_{1}, \vec{v}_{2}\right)
$$

where all second-order interactions of waves are included. In the course of this paper the interaction rate is usually presented as $\tilde{S}=|\vec{S}| /\left(\left|\vec{v}_{1}\right|\left|\vec{v}_{2}\right|\right)$, which is normalized such that the amplitude of the incident waves is assumed to be 1 . When changing to occupation numbers in the framework of quasiparticles, these numbers are defined as

$$
N=\frac{\mathfrak{E}}{\hbar \omega}
$$

where $\mathfrak{E}$ is the energy of the given wavemode. For occupation numbers $\vec{S}$ has to be transformed into the interaction rate $|V|^{2}$ (see Eq. I-26]sg). In order to do so, one define $N(k)=|c(k)|^{2}$, which yields

$$
\partial_{t} c_{0}=V c_{1} c_{2} \Rightarrow \partial_{t} N_{0} \propto|V|^{2} N_{1} N_{2}
$$

\section{Results}

The resonance conditions of the dispersive waves corresponding to the non-dispersive three-wave interactions, $A^{+} \leftrightarrow S^{+}+A^{-}$(Fig. 1), show very different behavior depending on the polarization. For the right-hand polarized waves (Fast-wave - Whistler mode), the interaction is qualitatively similar to the non-dispersive case: The frequency difference of the R-mode waves is small and increasing with increasing R-wave frequency. As a result of damping of the ion-sound mode (I-mode, which is used as dispersive equivalent to sound mode $\mathrm{S}$ ), the standard inverse cascade of $\mathrm{R}$-waves is produced as in the nondispersive case. However, the left-hand polarized waves (Alfvén - ion-cyclotron mode) behavior is very different: as the frequency of the mother wave approaches the resonance 


\section{Low $\beta$-Plasma}

$\mathrm{I}^{+}+\mathrm{R}^{-} \leftrightarrow \mathrm{R}^{+}$

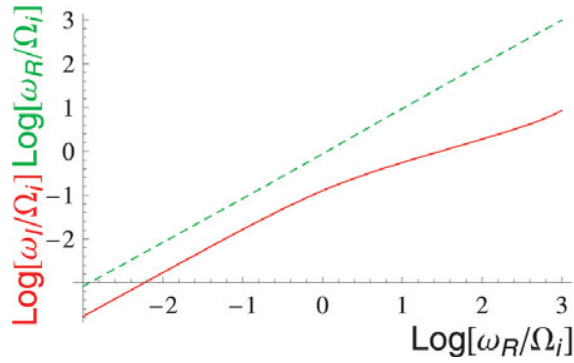

$\mathrm{I}^{+}+\mathrm{L}^{-} \leftrightarrow \mathrm{L}^{+}$

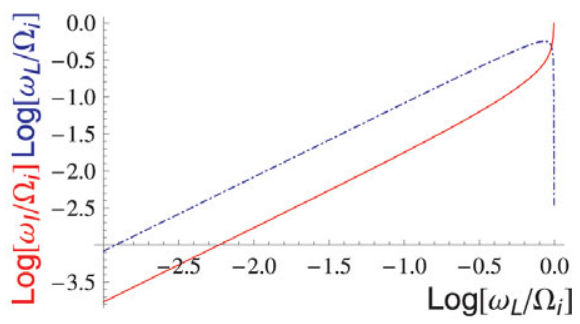

Figure 1. Resonance condition for the standard low- $\beta$ interactions which resemble the classical interaction $A^{+} \leftrightarrow S^{+}+A^{-}$. The green (dashed) curved represents the frequency of R-mode, blue (dashed-dotted) curves the L-mode and the red (solid) curve the I-mode respectively.
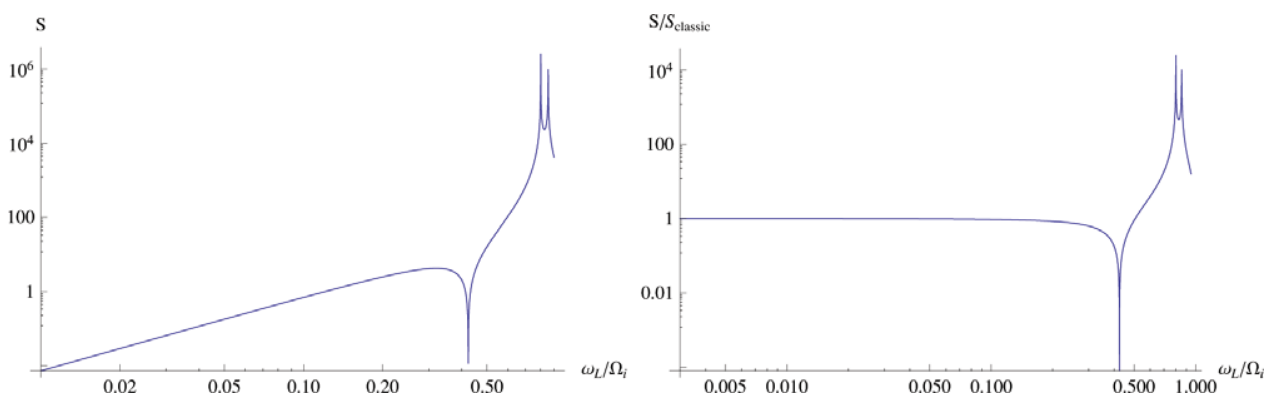

Figure 2. Interaction rate $c$ for the interaction $L^{+} \leftrightarrow I^{+}+L^{-}$and its comparison to the classical rate

at $\omega \rightarrow \Omega_{i}$, the daughter-wave frequency becomes very small. Thus, ion-cyclotron waves are able to decay directly into low-frequency Alfvén waves and at the same time emit high-frequency sound waves that can be easily damped through ion-Landau damping. Most of the energy of the ion-cyclotron waves is, thus, dissipated in the process.

While looking at the interaction rates $(\tilde{S})$, one notes that the R-mode interactions are again pretty similar to the non-dispersive case. The L-mode, on the other hand, experiences a couple of orders-of-magnitude increase in $\tilde{S}$ over the non-dispersive result in the frequency range $\omega \gtrsim 0.6 \Omega_{i}$, where the dispersion relation (and the three-wave resonance condition) is modified. This offers a dissipation channel for waves emitted through kinetic instabilities in this frequency range. Below the high-frequency range (around $\omega \sim 0.5 \Omega_{i}$ ), there is a short range of frequencies, where the interaction rate is small. However, this range of frequencies will be modified if the resonance takes account of the ${ }^{4}$ He resonance.

Thus, the inverse cascading of the $\mathrm{R}$ mode closely resembles the non-dispersive case, while the L mode interactions are strongly modified. Waves generated in the dispersive range, e.g., through kinetic instabilities involving electron beams, will be cascaded down to lower frequencies through very different channels depending on their polarization. 

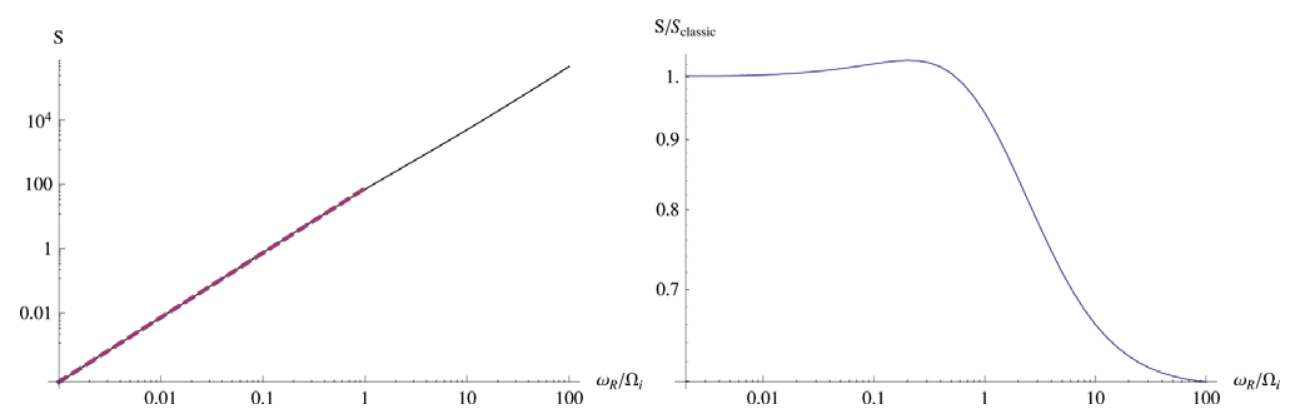

Figure 3. Interaction rate $c$ for the interaction $R^{+} \leftrightarrow I^{+}+R^{-}$and its comparison to the classical rate

\section{Discussion and Conclusions}

In this paper an extension of three-wave interactions to the dispersive limit is discussed. The possible interactions which are derived from the dispersion relations of L- and Rwaves differ with the wave polarization. The present study shows the expected difference of L- and R-modes around the gyrofrequency. While the interaction of R-waves closely resembles the non-dispersive, the L-wave interaction show strong dispersion effects close to the ion-gyrofrequency. This can also be seen in the interaction rate which is derived from Hall-MHD. This modification of L-wave interactions has an influence on the heating in coronal holes.

Wave heating models based on the cyclotron damping mechanism have problems in explaining the proton temperatures see e.g.]mvh, as cyclotron resonance is realized first for minor ions and alphas. The non-linear mechanism relying on the Landau damping of the ions sound waves, however, has the attractive feature that all ions with thermal speeds comparable to the ion sound speeds can Landau damp the waves. As electrons have much higher thermal speeds, their Landau damping rates are smaller than those of the ions.

The presented model is not complete in the sense that only interactions are discussed which resemble classic (non-dispersive) interactions. A full model of non-linear interactions in the solar corona requires also dispersive-only interactions. This model combined with appropriate damping rates would then allow for an accurate prediction of turbulence power spectra.

Due to the obvious difference in the decay of L- and R-modes, which in turn leads to a faster damping of L-modes, one may expect to see changes in the magnetic helicity of the system in the dissipation range $\left(k>\Omega_{i} / v_{A}\right)$.

\section{References}

Axford, W. I. \& McKenzie, J. F. 1992, Solar Wind Seven, 1

Chin, Y.-C. \& Wentzel, D. G. 1972, Ap\&SS, 16, 465

Laitinen, T., Fichtner, H., \& Vainio, R. 2003, JGR (Space Physics), 108, 1081

Markovskii, S. A., Vasquez, B. J., \& Hollweg, J. V. 2009 ApJ, 695, 1413

Melrose, D. B. \& Sy, W. 1972, ApधSSS, 17, 343

Sagdeev, R. Z. \& Galeev, A. A. 1969 Nonlinear Plasma theory

Spanier, F. \& Vainio, R. 2009, ASL, 2, 337

Vainio, R. \& Spanier, F. 2005, A\&A, 437, 1

Yoon, P. H. \& Fang, T.-M. 2009 PPCF, 50, 8 